\title{
Informing decision-making for universal access to quality tuberculosis diagnosis in India: an economic-epidemiological model
}

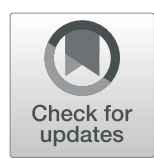

\author{
Hojoon Sohn ${ }^{1 *}\left(\mathbb{D}\right.$, Parastu Kasaie ${ }^{1 \dagger}$, Emily Kendall ${ }^{2}$, Gabriela B. Gomez ${ }^{3}$, Anna Vassall ${ }^{3}$, Madhukar Pai ${ }^{4,5}$ and \\ David Dowdy ${ }^{1}$
}

\begin{abstract}
Background: India and many other high-burden countries have committed to providing universal access to highquality diagnosis and drug susceptibility testing (DST) for tuberculosis (TB), but the most cost-effective approach to achieve this goal remains uncertain. Centralized testing at district-level hub facilities with a supporting sample transport network can generate economies of scale, but decentralization to the peripheral level may provide faster diagnosis and reduce losses to follow-up (LTFU).

Methods: We generated functions to evaluate the costs of centralized and decentralized molecular testing for tuberculosis with Xpert MTB/RIF (Xpert), a WHO-endorsed test which can be performed at centralized and decentralized levels. We merged the cost estimates with an agent-based simulation of TB transmission in a hypothetical representative region in India to assess the impact and cost-effectiveness of each strategy.

Results: Compared against centralized Xpert testing, decentralization was most favorable when testing volume at decentralized facilities and pre-treatment LTFU were high, and specimen transport network was exclusively established for TB. Assuming equal quality of centralized and decentralized testing, decentralization was cost-saving, saving a median $\$ 338,000$ (interquartile simulation range [IQR] - $\$ 222,000 ; \$ 889,000$ ) per 20 million people over 10 years, in the most cost-favorable scenario. In the most cost-unfavorable scenario, decentralized testing would cost a median \$3161 [IQR \$2412; \$4731] per disability-adjusted life year averted relative to centralized testing.

Conclusions: Decentralization of Xpert testing is likely to be cost-saving or cost-effective in most settings to which these simulation results might generalize. More decentralized testing is more cost-effective in settings with moderate-to-high peripheral testing volumes, high existing clinical LTFU, inability to share specimen transport costs with other disease entities, and ability to ensure high-quality peripheral Xpert testing. Decision-makers should assess these factors when deciding whether to decentralize molecular testing for tuberculosis.
\end{abstract}

Keywords: Tuberculosis, Diagnostic techniques and procedures, Cost-benefit analysis, Systems analysis

\section{Introduction}

Tuberculosis (TB) is one of the world's most important infectious causes of morbidity and mortality, with an estimated $25 \%$ of the world's population infected [1] and 1.6 million deaths reported in 2017 [2]. Global TB incidence is declining slowly ( $2 \%$ per year), far short of the

\footnotetext{
* Correspondence: hsohn6@jhu.edu

${ }^{\dagger}$ Hojoon Sohn and Parastu Kasaie contributed equally to this work.

1 Department of Epidemiology, Johns Hopkins Bloomberg School of Public

Health, 615 N. Wolfe St., E6529, Baltimore, MD 21205, USA

Full list of author information is available at the end of the article
}

progress needed to achieve global targets [2]. With only $64 \%$ of an estimated 10 million new cases reported in 2017 [2], strategies to optimize existing technologies and interventions to address gaps in $\mathrm{TB}$ case detection and linkage to care are desperately needed.

Xpert MTB/RIF (Xpert), a cartridge-based nucleic acid amplification test (CBNAAT) for TB with substantially improved sensitivity relative to sputum smear microscopy (SSM) [3], has been recommended by the World Health Organization (WHO) [4] and scaled up in many settings worldwide. Many studies have suggested that

(c) The Author(s). 2019 Open Access This article is distributed under the terms of the Creative Commons Attribution 4.0 International License (http://creativecommons.org/licenses/by/4.0/), which permits unrestricted use, distribution, and reproduction in any medium, provided you give appropriate credit to the original author(s) and the source, provide a link to the Creative Commons license, and indicate if changes were made. The Creative Commons Public Domain Dedication waiver (http://creativecommons.org/publicdomain/zero/1.0/) applies to the data made available in this article, unless otherwise stated. 
Xpert is both feasible and cost-effective [5-7] in idealized settings. Nevertheless, in India-as in many resource-limited settings-the use of Xpert has largely been limited to the public sector (at district level or higher [8]) and often restricted to high-priority populations including HIV-positive individuals, children, and people at high risk for multidrug-resistant (MDR) TB [9]. As a result, in more peripheral settings where the majority of public-sector patients present for care (e.g., primary care clinics), TB diagnosis continues to rely on SSM and non-microbiological tests.

Since the initial development of the GeneXpert system, much progress has been made in the development of smaller point-of-care (POC) instruments. In 2018, the battery-operated, single-module GeneXpert Edge system was introduced, and the point-of-care GeneXpert Omni system is expected to be released in 2020. As such, decentralization of Xpert at peripheral facilities using these POC platforms may help address the problem of pre-treatment loss to follow-up (LTFU), which is estimated to occur among $13 \%$ of individuals with confirmed TB in Asia [10]. An alternative to decentralization is to centralize Xpert testing at district-level or reference facilities and establish a "hub-and-spoke" transport network for clinical specimens (e.g., sputum) [11]. This approach could enable cost sharing across disease entities, consolidate maintenance of high-level infrastructure in more centralized facilities, and ensure higher testing volume to achieve economies of scale. South Africa, for example, has successfully used this centralized model via their National Health Laboratory Service (NHLS) [12].

Given the resource constraints and the complexity and heterogeneity of most healthcare systems, it is important to estimate the impact and cost-effectiveness of more centralized Xpert testing versus decentralizing Xpert testing capacity to more peripheral settings. This is also a question of generalizable importance, as many other tests on the WHO Essential Diagnostics List (e.g., HIV viral load testing, glycated hemoglobin measurement for diabetes) could be performed either centrally or in point-of-care fashion [13]. To help illustrate the primary considerations in evaluating the cost and cost-effectiveness of more decentralized versus more centralized Xpert testing, we constructed a suite of economic and epidemiological models in a hypothetical setting representative of the Indian public sector.

\section{Methods}

\section{Experimental framework}

Our primary comparison was between more centralized Xpert testing for TB at district/city-level hubs with supporting sample transport networks ("centralized" scenario) and decentralization of Xpert testing to more peripheral settings in clinics where microscopy currently is performed ("decentralized" scenario) within India's current public sector system, namely the Revised National Tuberculosis Control Program (RNTCP) [14]. In the decentralized scenario, we assumed that Xpert is made available (for example, using battery-operated Edge instruments, or Omni, in the near future) at the Microscopy Center (MC), a primary health center serving approximately 100,000 people with a capacity to perform SSM. In this scenario, we assumed a sufficient number of instruments installed to ensure the same-day diagnosis of $>90 \%$ of all patients with presumptive TB.

In the centralized scenario, we assumed that Xpert is available only at the District TB Center (DTC), a central referral facility currently equipped with four-module GeneXpert IV instruments that cannot readily be implemented at the point of care. There are more than 13,000 MCs in India, with a median of 13 MCs (interquartile range $(I Q R)=9-23)$ associated per DTC. We assumed that sputum specimens are transported from individual MCs to the DTC using a specimen referral network. In both decentralized and centralized scenarios, we consider only patients who present to peripheral centers (i.e., MCs) for testing, as decentralization will not affect outcomes of patients presenting directly to district-level centers (i.e., DTCs) for diagnosis. In both scenarios, we assumed that $13 \%$ of individuals presenting to care would experience LTFU prior to treatment initiation and experience no benefit from TB testing (Fig. 1, red arrow). These individuals may undergo other diagnostic encounters, but the cost and outcome of those encounters are assumed not to differ between scenarios (and thus not explicitly considered) [10]. Since referral tests generally cannot provide same-day results, we assumed that centralized testing would result in additional pretreatment LTFU for some patients who would receive same-day treatment in the decentralized scenario (Fig. 1, blue arrow); we assume that these patients will return for follow-up visits and obtain an appropriate (though delayed) diagnosis in the future. In both strategies, Xpert was assumed to be used as the primary diagnostic tool for all presumptive TB patients, a key objective of India's 2025 National Strategic Plan to ensure universal and upfront access to molecular diagnostics for TB.

To evaluate cost-effectiveness, our a priori assumption was that decentralized testing would be more expensive (as it would require more equipment modules), but also more effective (as it would reduce pre-treatment LTFU). To evaluate this trade-off, we first constructed a range of cost functions to estimate the unit cost of Xpert from the health system perspective, under various scenarios. We then adapted a published agent-based simulation of drug-susceptible (DS) and drug-resistant (DR) TB transmission in India [15] to estimate the incremental epidemiological impact and cost-effectiveness (assessed 


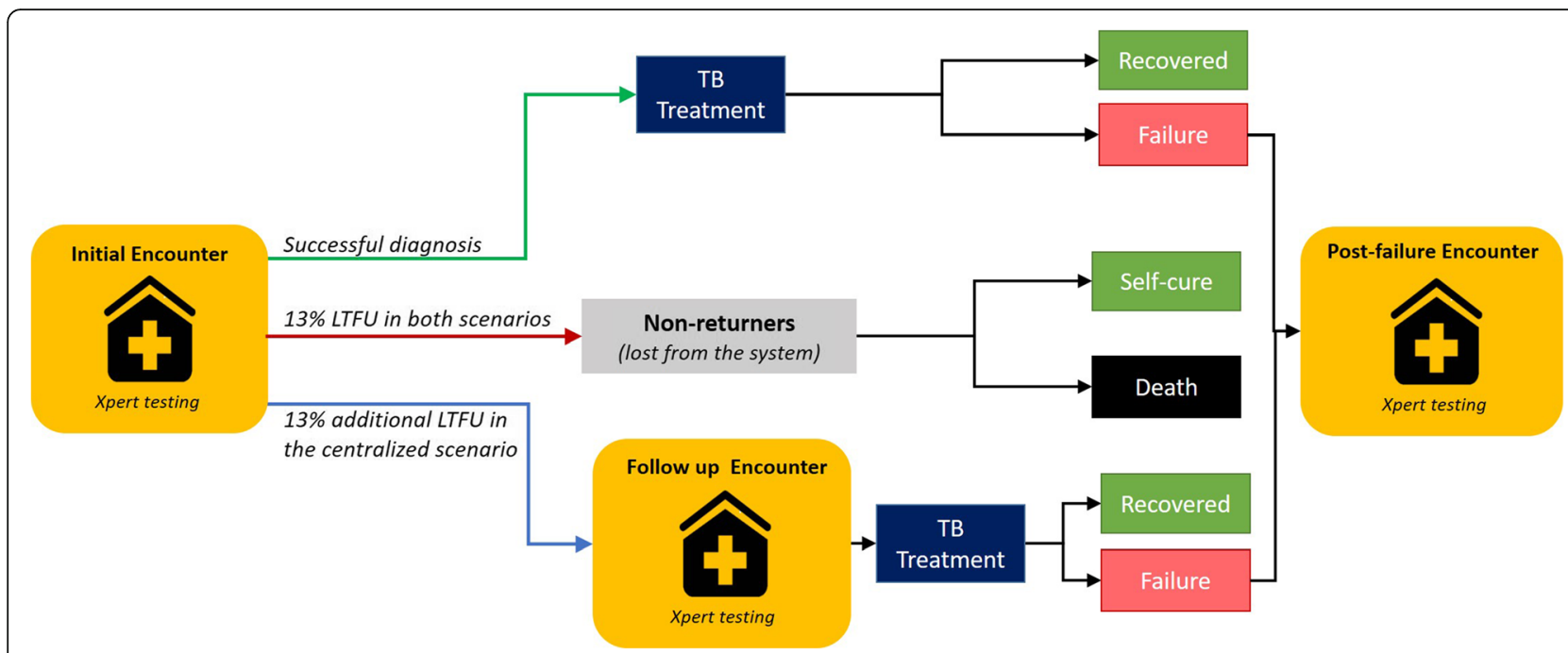

Fig. 1 Schematic representation of the tuberculosis (TB) diagnostic process in the public sector. Upon accessing the public sector for TB diagnosis, individuals are assumed to undergo an initial evaluation that includes Xpert MTB/RIF (or sputum smear microscopy prior to Xpert implementation). A proportion of those encounters will result in successful diagnosis followed by successful treatment (green arrow) or pretreatment loss to follow-up (LTFU) estimated at 13\% for both centralized and decentralized scenarios (red arrow). These individuals may undergo other diagnostic encounters, but those encounters do not result in TB diagnosis, and the cost of those encounters is not considered by the model. In addition to these individuals, another proportion of individuals (also estimated at 13\% in the base case) are expected to experience pre-treatment LTFU due to diagnostic delays in the centralized scenario (blue arrow). These individuals can return for a future visit in which Xpert is again performed and TB treatment is successfully initiated. The assumption that all incremental LTFU is followed by a second encounter is conservative and may underestimate the impact of Xpert in the decentralized scenario. Finally, individuals receiving TB treatment who fail to recover will undergo a separate encounter after treatment completion in which Xpert is performed and used to guide second-line therapy

as the cost per disability-adjusted life year [DALY] averted) of decentralized versus centralized Xpert.

\section{Cost model}

Using a bottom-up micro-costing method incorporating data from our previous work in India $[5,16]$, we constructed a reference per-test cost table for Xpert (see Additional file 1: Section S1.2). We estimated the price of a peripheral single-module GeneXpert unit as US\$2895, with similar per-module maintenance pricing as the GeneXpert IV [17]. We inflated all Xpert-related (equipment and cartridge) unit prices by $20 \%$ to account for procurement and installation. We assumed that the amount of time required to perform an Xpert test did not differ with centralized versus decentralized testing, as the procedures are standardized, require minimal hands-on time, do not differ based on the type of GeneXpert system used, and can be operated by existing laboratory staff (e.g., microscopy technicians who currently perform smear testing at MCs). We also assumed the equivalent quality of testing, as decentralized testing is unlikely to be accepted in settings where quality is substantially compromised. We therefore incorporated costs of continuous quality control measures under each strategy.

Anticipating that laboratory workload (number of tests per day) would strongly influence the unit cost of Xpert
$[5,18]$, we simulated a range of daily testing volumes for MCs and DTCs assuming a Poisson distribution of tests per day and 250 annual operational days. Mean per-test cost was assessed for each volume scenario based on annual total cost and testing volume (see Additional file 1: Section S1.3).

For decentralized testing, we evaluated 15 different scenarios (Fig. 2) with mean daily testing volumes between 0.1 (i.e., 25 samples tested per year) and ten (2500 samples/year). This range covers the expected mean daily workload of 3-6 tests per MC nationwide [14]. For centralized testing, a total of seven mean daily testing volume scenarios between 10 (2475 samples/year) and $70+$ (17,411 samples/year) were assessed, including costs associated with sample transport (see Additional file 1: Section S1.4). At the time of analysis, no published evidence on costs of establishing or operating sample transport networks for sputum specimens was available, to our knowledge. Therefore, we estimated mean persample transport costs based on the following: (a) distributions of MC-to-DTC ratio and mean distance from MCs to DTC (based on total service area of DTC/number of $\mathrm{MC}$ in the area) in India (see Additional file 1: Figure S2), (b) hypothetical estimates of the number of specimens transported, and (c) a range of cost sharing of sample transport (5 to 100\%) with other clinical specimens (Additional file 1: Table S4). This 


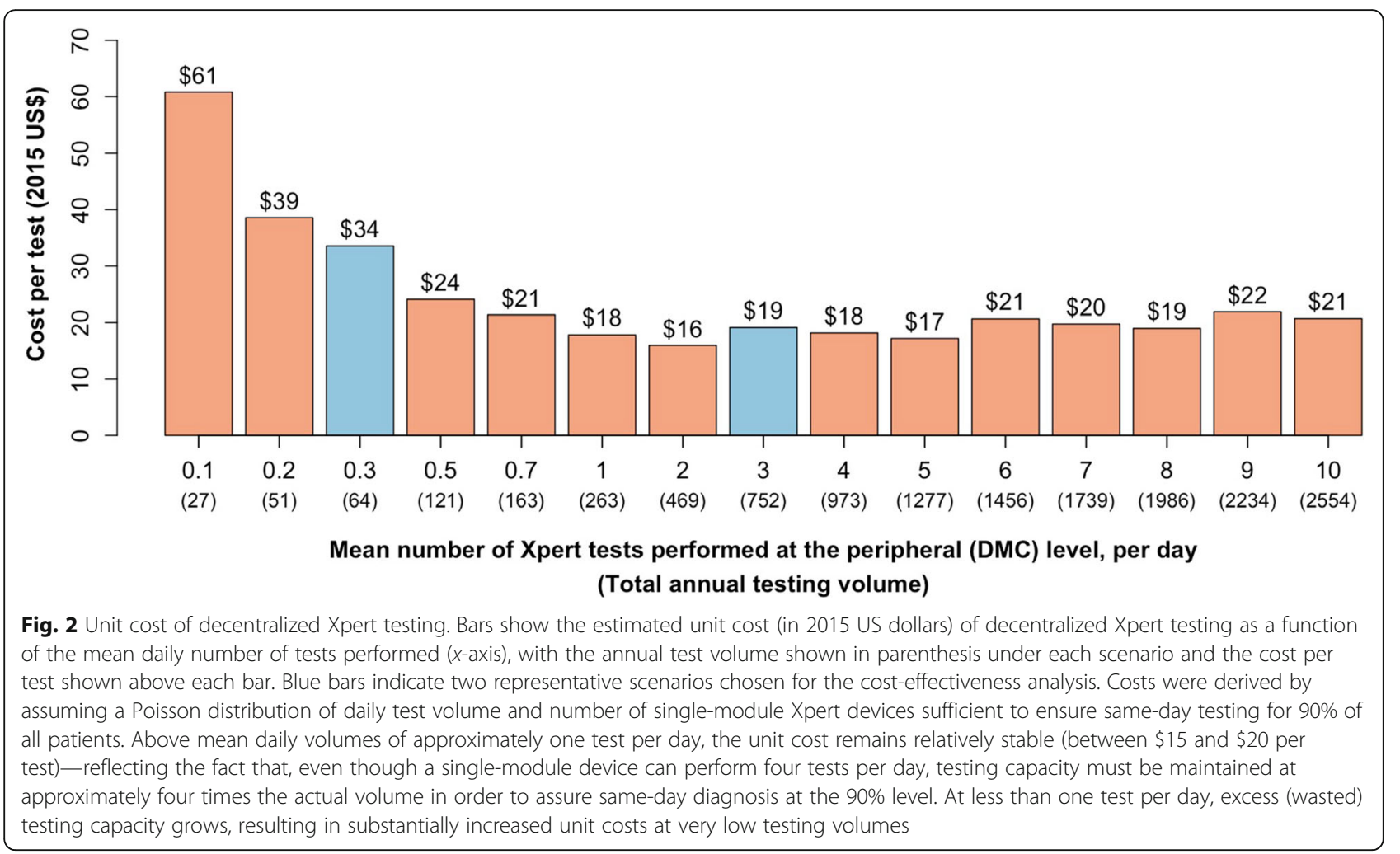

cost was calculated separately for each DTC test volume scenario (Additional file 1: Table S6).

Costs for clinical encounters (outpatient visits, clinical evaluation, antibiotic treatment, etc.), TB-specific treatment, and chest X-ray were estimated from sources including the Global Drug Facility, the MSH International Price Tracker, the World Health Organization's CHOosing Interventions that are Cost-Effective (WHOCHOICE) database [19], and published literature (Table 1) [22, 23]. Full details of the costing approach are described in Additional file 1: Section S1.

\section{Agent-based simulation model}

We developed an agent-based simulation model of TB transmission and treatment in India, incorporating household structure, patients' care-seeking pathways, and the public-sector TB care cascade (Fig. 1, Additional file 1: Figures S4 and S5). A detailed description of the model is presented in Additional file 1: Section S2 and earlier publications [15, 20, 21, 24]. Briefly, the simulation model was calibrated to 2017 WHO estimates of TB incidence, prevalence, and mortality in India using a stepwise procedure (see Additional file 1: Section S2.5). At the end of the calibration period, we modeled the introduction of Xpert in area(s) where Xpert testing is not available, as a first-line test replacing on-site SSM, under decentralized and centralized testing scenarios as described above. The model was then run for 10 years to project corresponding changes in the incidence and mortality of DS- and DR-TB. The core model simulates a population of one million people, representative of an average-sized district in India that might contain a single DTC [25]. For each Xpert testing scenario (centralized versus decentralized), we generated 2000 independent simulations of TB transmission at the district level. To assess the policy implementations at a city or state level, we then constructed larger simulated populations of 20 million people by combining 20 randomly sampled districts (with replacement) from the original pool of simulations. We repeated this procedure to achieve a sample of 2000 larger-scale simulations with a population of 20 million individuals each. All outcomes were reported as median values with interquartile uncertainty ranges across these 2000 larger-scale simulated populations.

\section{Cost-effectiveness analysis}

Given our a priori assumption that decentralized testing would be implemented with equal quality and would reduce pre-treatment LTFU with no adverse consequences (i.e., that decentralized testing would be more effective than centralized testing and thus preferred in settings where decentralized testing was less costly), we focused our cost-effectiveness analyses on our tested scenarios where the unit cost of decentralized testing would be higher than that of decentralized testing. We selected 
Table 1 Selected model parameters for post-calibration sensitivity analysis

\begin{tabular}{|c|c|c|c|c|}
\hline Parameters & Base value & Low bound ${ }^{+}$ & High bound & Reference \\
\hline \multicolumn{5}{|l|}{ Xpert diagnostic accuracy } \\
\hline Sensitivity—smear positive & 0.98 & 0.93 & 1.00 & \multirow[t]{5}{*}{ [3] } \\
\hline Sensitivity_-smear negative & 0.67 & 0.60 & 0.85 & \\
\hline Sensitivity—RIF resistance & 0.95 & 0.90 & 0.97 & \\
\hline Specificity_diagnosis of TB disease & 0.99 & 0.98 & 1.00 & \\
\hline Specificity—RIF resistance & 0.98 & 0.97 & 1.00 & \\
\hline \multicolumn{5}{|l|}{ Treatment and linkage to care } \\
\hline Probability of empiric treatment at initial encounter & 0.25 & 0.00 & 0.50 & Assumption \\
\hline Probability of loss to follow-up after initial encounter & 0.13 & 0.05 & 0.25 & {$[10]$} \\
\hline Monthly rate of returning for follow-up encounter & 0.46 & 0.30 & 0.70 & {$[20]$} \\
\hline Probability of treatment failure for drug-resistant TB & 0.17 & 0.10 & 0.25 & WHO 2016 \\
\hline Probability of referring from private provider to public sector & 0.67 & 0.54 & 0.80 & {$[21]$} \\
\hline \multicolumn{5}{|l|}{ Type of costs } \\
\hline Cost of initial encounter & $\$ 12.11$ & $\$ 9.08$ & $\$ 15.14$ & \multirow[t]{6}{*}{ Recalculated based on $[19,22,23]$} \\
\hline Cost of follow-up encounter & $\$ 9.53$ & $\$ 7.15$ & $\$ 11.91$ & \\
\hline Cost of encounter to assess failing treatment & $\$ 7.39$ & $\$ 5.54$ & $\$ 9.24$ & \\
\hline Other visits (informal/private) & $\$ 2.32$ & $\$ 2.06$ & $\$ 2.60$ & \\
\hline DS-TB treatment, per 6-month course & $\$ 345.65$ & $\$ 259.24$ & $\$ 432.06$ & \\
\hline DR-TB treatment, per course & $\$ 3399.22$ & $\$ 2549.41$ & $\$ 4249.02$ & \\
\hline
\end{tabular}

${ }^{+}$One-way sensitivity analysis was performed by varying each parameter in this table individually between its low and high bounds. For a list of all parameters and references, see Section S2.6 of Additional file 1

these scenarios based on their conceptual importance: one scenario of very low decentralized test volumes $(0.3$ tests per day, as might be seen in more sparsely populated districts, or as TB incidence declines) and one of average test volumes (three per day, reflective of mean MC volumes in India) [14]. We compared these decentralized scenarios against centralized testing at high volume ( $>70$ tests per day) with either low or high levels of shared transport costs (see Fig. 3).

All analyses were performed from the health system perspective and assessed in 2015 US dollars. The primary outcome was calculated as the ratio of incremental costs per disability-adjusted life year (DALY) averted over a 10-year period, with costs and DALYs discounted at $3 \%$ annually. We benchmarked cost-effectiveness against a range of cost-effectiveness thresholds (CET) from $\$ 500$ to $\$ 2000$ to reflect more realistic local constraints than reflected in traditional per capita Gross Domestic Product (GDP)-based thresholds [26, 27].

\section{Sensitivity analyses}

We performed univariate sensitivity analyses for selected epidemiological and cost-related parameters across the ranges shown in Table 1. For each analysis, we evaluated the relative epidemiological impact (reduction in DS-TB incidence), difference in cost, and incremental cost-effectiveness, comparing decentralized to centralized testing. For these analyses, we assumed a decentralized scenario with moderate volume of three tests per day and a centralized scenario with no cost sharing for sample transport. We conducted other sensitivity analyses in which we separately varied incremental pre-treatment LTFU in the centralized scenario, diagnostic accuracy of Xpert, and other drivers of the unit cost per Xpert test (e.g., estimated useful life).

\section{Results}

The unit cost of decentralized Xpert ranged from $\$ 17.20$ to $\$ 60.81$ (Fig. 2), but consistently approximated \$20 per test when MCs performed at least one test a day (with small fluctuations reflecting the number of testing modules required). The unit cost of centralized Xpert depended on the degree to which the costs of sample transport could be shared with clinical specimens for non-TB disease entities (e.g., from $\$ 14.00$ to $\$ 18.96$ in the highest-volume scenario in Additional file 1: Table S8).

The per-test cost difference between centralized and decentralized testing was driven primarily by three factors: centralized (DTC) testing volume, cross-disease cost sharing of specimen transport under centralized testing (between 5 and $100 \%$ of the sample transport network dedicated for $\mathrm{TB} /$ sputum specimens), and peripheral $(\mathrm{MC})$ testing volume. At higher decentralized 


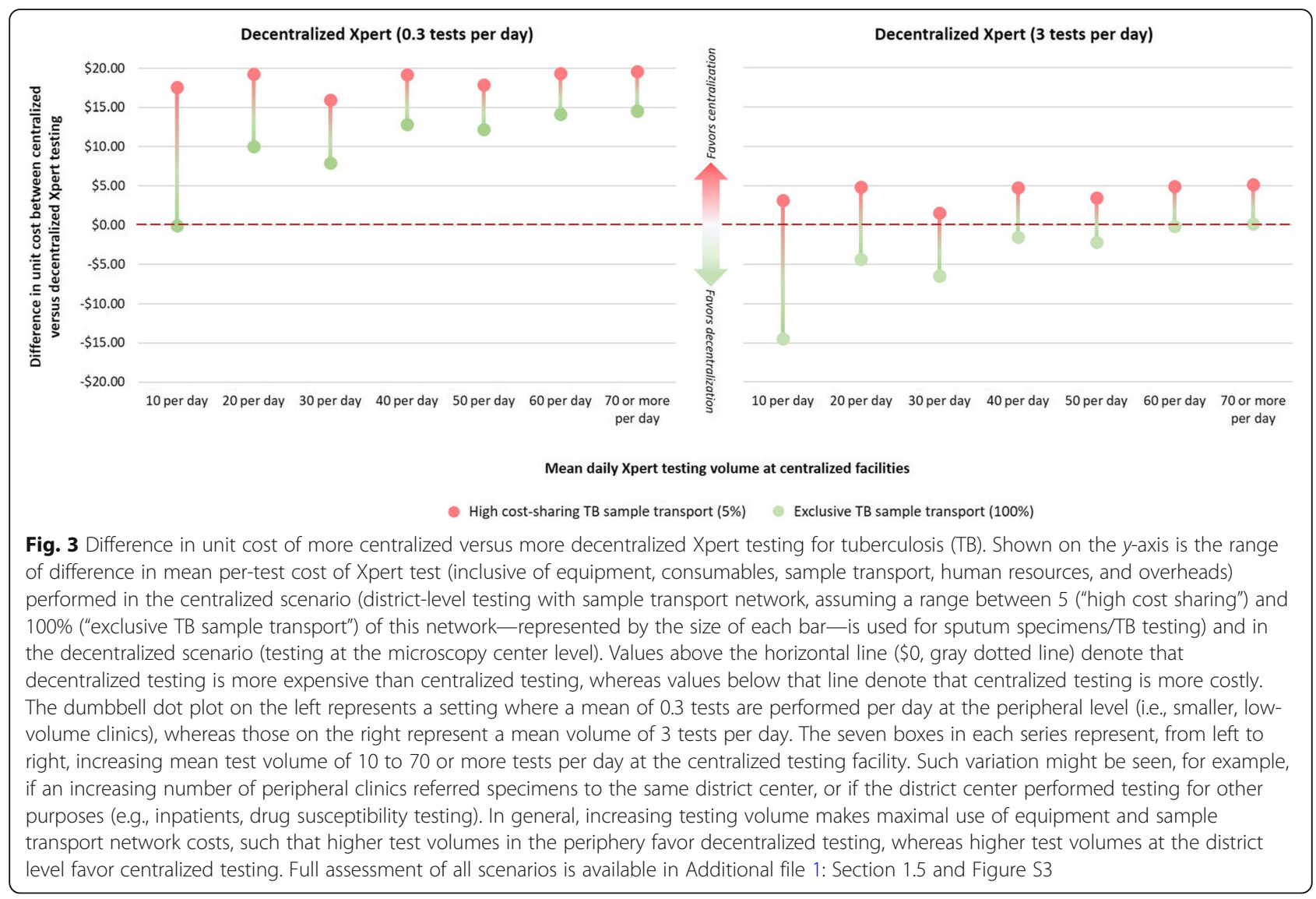

testing volume (mean daily testing volume of 3 or more at MCs, represented by $\lambda=3$ ), differences in costs of between centralized and decentralized testing (Fig. 3) were generally small or favored decentralized testing (as indicated by a negative cost estimate). At lower decentralized testing volumes (MC mean daily testing volume of less than 1 test, for example, $\lambda=0.3$ ), centralized testing was generally cheaper than decentralized testing. In both cases, higher sample transport cost sharing and testing volumes for centralized testing lowered the unit cost of centralized testing. Additional description of the calculation of unit costs and sensitivity analyses around the drivers of these costs are shown in Additional file 1: Section S1.6.

\section{Epidemiological impact of decentralized and centralized Xpert}

Assuming that centralized Xpert was used to replace SSM starting in 2018 (with 13\% pre-treatment LTFU relative to decentralized testing [10]), our model projected a median TB incidence of 213 [IQR 212-214] cases per 100,000 in 2028 in a hypothetical region representative of India's public sector where Xpert testing was previously unavailable. Alternatively, if Xpert was implemented in decentralized fashion (with equal quality and no pre-treatment LTFU) in the same region, the resulting DS-TB incidence in 2028 was projected to be $1.0 \%$ [IQR $0.5-1.6 \%$ ] lower than with centralized testing, equivalent to averting a total of 15 [IQR 4-27] new DSTB cases per 100,000 population over 10 years (Fig. 4a). For DR-TB incidence and TB mortality, the difference between the two strategies was small, with decentralized testing averting an estimated 1 [IQR -1 to 5 ] incident DR-TB case and 4 [IQR 0-9] total TB deaths per 100, 000 over 10 years (Fig. 4b, c).

\section{Incremental cost-effectiveness of decentralized versus centralized Xpert}

Primary cost-effectiveness results are given in Table 2 and Fig. 5. In the primary analysis, decentralized Xpert averted an estimated $1.25 \%$ [IQR $0.88 \%, 1.54 \%$ ] of all TB-related DALYs, relative to centralized Xpert. Under the most unfavorable scenario for decentralized testing (low decentralized volume at 0.3 tests per day and 95\% cost sharing for specimen transport in the centralized scenario), this gain came at an estimated cost of \$3161 [IQR \$2412; \$4731] per DALY averted. Under the most favorable scenario (high decentralized volume at 3 tests per day and no cost sharing), decentralized testing was cost saving compared to centralized testing, saving a 



Fig. 4 Projected incremental epidemiological impact of decentralized versus centralized Xpert testing for tuberculosis (TB). Panels compare the impact of Xpert implemented via a decentralized strategy compared to centralized testing, in terms of the cumulative number of DS-TB infections averted (a), cumulative number of drug-resistant (DR) TB infections averted (b), and cumulative number of TB deaths averted (c). All values are adjusted to a population size of 100,000 . Shaded areas and arrows represent interquartile uncertainty ranges

median of $\$ 338,000[\mathrm{IQR}-\$ 222,000 ; \$ 889,000]$ per 20 million people.

The probability that decentralized Xpert would be cost-effective depended strongly on decentralized testing volume, degree of cost sharing for centralized specimen transport, and the cost-effectiveness threshold. For example, the probability that decentralized testing would be cost-effective relative to centralized testing ranged from 0.12 to 0.98 at a willingness to pay of $\$ 2000$ per DALY averted (Fig. 5). At very low peripheral testing volumes ( $\leq 0.3$ tests per day), decentralized Xpert was unlikely to be cost-effective: even at a willingness to pay of $\$ 6000$ per DALY averted, the probability of cost-effectiveness remained below 0.8 .

\section{Sensitivity analyses}

When comparing decentralized against centralized testing, differences in outcome measures-epidemiological impact of Xpert (Fig. 6a), costs (Fig. 6b), and DALYs (Fig. 6c)were generally robust to one-way variation in parameter values. Incremental cost-effectiveness ratios (Fig. 6d) likewise did not vary substantially in one-way sensitivity analyses, with the uncertainty boundary remaining within $\pm 10 \%$ of the primary estimate. Increasing the amount of

Table 2 Incremental cost-effectiveness of decentralized versus centralized Xpert testing

\begin{tabular}{|c|c|c|c|c|}
\hline & $\begin{array}{l}\text { Centralized Xpert } \\
\text { with } 95 \% \text { cost } \\
\text { sharing for specimen } \\
\text { transport }\end{array}$ & $\begin{array}{l}\text { Centralized Xpert with no cost sharing } \\
\text { for specimen transport }\end{array}$ & $\begin{array}{l}\text { Decentralized Xpert with } \\
\text { test volume at } 3 \text { per day }\end{array}$ & $\begin{array}{l}\text { Decentralized Xpert with test } \\
\text { volume at } 0.3 \text { per day }\end{array}$ \\
\hline $\begin{array}{l}\text { Total costs (2015 US\$, in } \\
\text { million) }\end{array}$ & $\$ 189[\$ 157-\$ 222]$ & $\$ 196[\$ 164-\$ 230]$ & $\$ 196[\$ 164-\$ 230]$ & $\$ 217[\$ 185-\$ 253]$ \\
\hline Total DALYs & \multicolumn{2}{|c|}{$740,124[700,676-782,488]$} & \multicolumn{2}{|l|}{$730,923[692,001-773,630]$} \\
\hline \multicolumn{5}{|c|}{ Comparing centralized vs. decentralized Xpert } \\
\hline Centralized Xpert & $\begin{array}{l}\text { vs. Decentralized } \\
\text { Xpert }\end{array}$ & Difference in costs (2015 US\$, in million) & Difference in DALYs & $\begin{array}{l}\text { Cost per DALY averted (2015 } \\
\text { US\$) }\end{array}$ \\
\hline $\begin{array}{l}95 \% \text { cost sharing for } \\
\text { specimen transport }\end{array}$ & $\begin{array}{l}\text { vs. Test volume at } 3 \\
\text { per day }\end{array}$ & $\$ 7[\$ 6.6-\$ 8]$ & $9.26[6231-12,112]$ & $\$ 795[\$ 573-\$ 1213]$ \\
\hline $\begin{array}{l}\text { No cost sharing for } \\
\text { specimen transport }\end{array}$ & $\begin{array}{l}\text { vs. Test volume at } 3 \\
\text { per day }\end{array}$ & $-\$ 0.33[-\$ 0.89$ to $\$ 0.22]$ & & $\begin{array}{l}\text { Dominated }^{*} \\
\left.\text { [dominated }^{*}-\$ 29\right]\end{array}$ \\
\hline $\begin{array}{l}95 \% \text { cost sharing for } \\
\text { specimen transport }\end{array}$ & $\begin{array}{l}\text { vs. Test volume at } \\
0.3 \text { per day }\end{array}$ & $\$ 28[\$ 27-\$ 30]$ & & $\$ 3161[\$ 2412-\$ 4731]$ \\
\hline $\begin{array}{l}\text { No cost sharing for } \\
\text { specimen transport }\end{array}$ & $\begin{array}{l}\text { vs. Test volume at } \\
0.3 \text { per day }\end{array}$ & $\$ 21[\$ 20-\$ 22]$ & & $\$ 2339[\$ 1775-\$ 3501]$ \\
\hline
\end{tabular}

A total of four cost sets were compared in the cost-effectiveness analysis. All values are expressed as median with interquartile ranges in brackets, cumulative and discounted over a 10-year time horizon

*In the median simulations, decentralized Xpert was less costly and more effective than centralized testing 


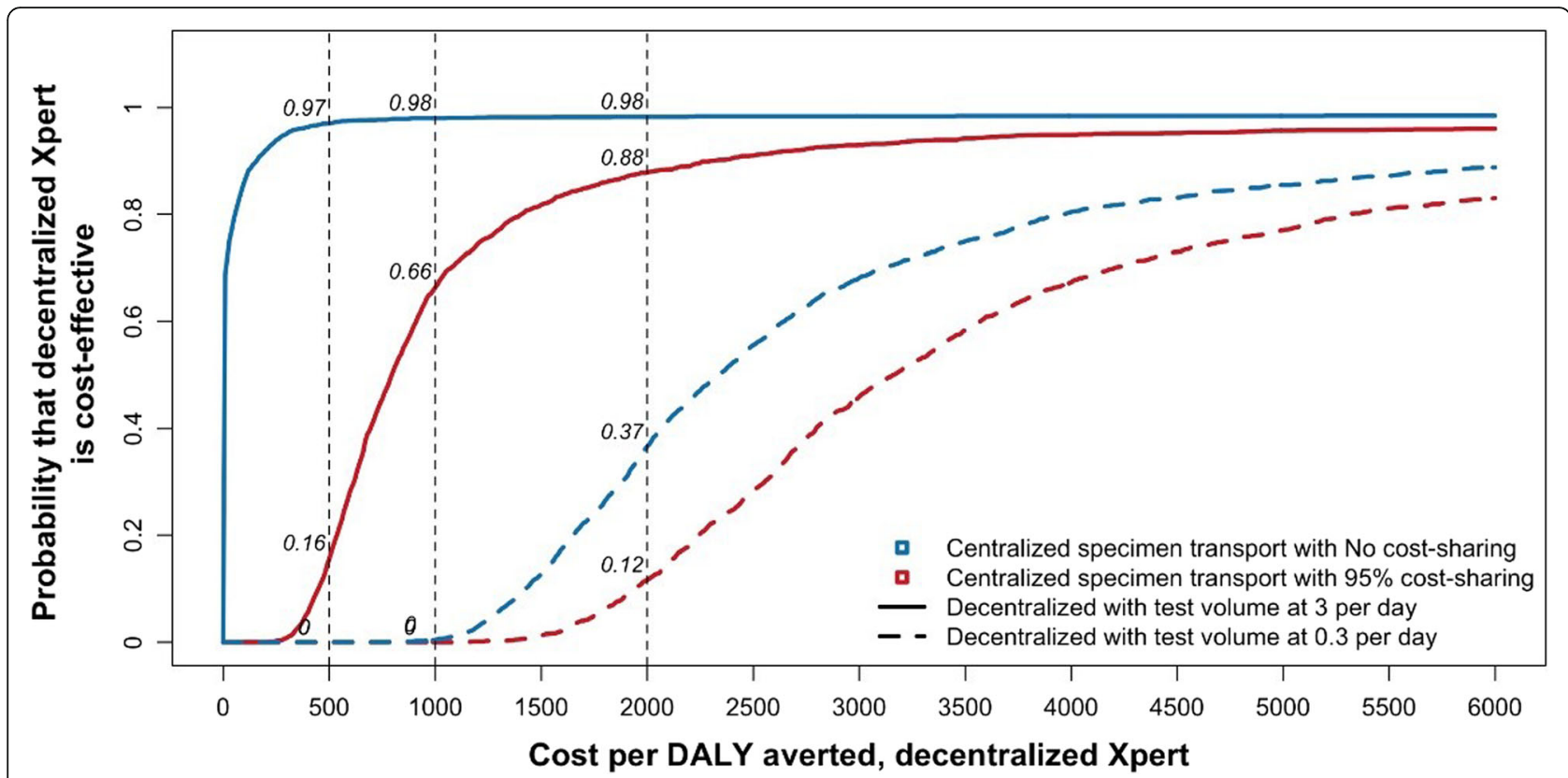

Fig. 5 Cost-effectiveness acceptability curves comparing decentralized versus centralized Xpert testing for tuberculosis (TB) in a representative simulated Indian population. The $x$-axis shows the cost per disability-adjusted life year (DALY) averted via decentralized Xpert compared to centralized testing, and the $y$-axis shows the proportion of stochastic simulations falling below each corresponding cost-effectiveness threshold. Dotted vertical lines represent alternative thresholds for evaluating cost-effectiveness. For each threshold, the cost-effectiveness values are computed for the four unit-cost comparisons when centralized specimen transport is utilized at no vs. 95\% cost sharing and mean peripheral testing volume ranges from 0.3 to 3 per day in the decentralized scenario

pre-treatment LTFU in the centralized scenario reduced the epidemiological impact of Xpert and resulted in higher costs and less favorable cost-effectiveness. For example, when increasing the incremental probability of pre-treatment LTFU with centralized (versus decentralized) testing from 0 to $20 \%$, the proportion of simulations favoring decentralized Xpert (assuming moderate decentralized volume, 95\% centralized cost sharing, and a willingnessto-pay threshold of \$1000 per DALY averted) rose from 57 to $99 \%$. Complete details of these analyses are given in Additional file 1: Sections S3.1 and S3.2.

Performing SSM as a back-up test in addition to Xpert (thereby allowing same-day diagnosis for all smear-positive patients) improved the epidemiological impact and cost-effectiveness of centralized testing. For example, assuming moderate decentralized testing volume and no cost-sharing for specimen transport, the probability that decentralized testing would be cost-effective relative to centralized testing was estimated as 0.61 (compared to 0.98 at baseline). For complete one-way sensitivity analyses results, please see Additional file 1, Section S3.3.

\section{Discussion}

This economic-epidemiological model of Xpert implementation in India suggests that the economics of decentralizing molecular testing for TB likely depend on three important factors: the testing volume at decentralized facilities and centralized facilities (i.e., how many decentralized facilities are referring), the degree to which costs of specimen transport can be shared with other non-TB disease entities, and the level of pre-treatment LTFU caused by the delays in centralized testing. Provided that decentralized testing can be performed with equal quality as centralized testing, the costs and LTFU incurred by using a hub-and-spoke system are likely to justify the increased costs of decentralized testing in most scenarios, except in settings where testing volumes in the periphery remain very low (mean $<1$ test per day).

Our results may appear contrary to existing arguments that point-of-care diagnosis is much more expensive than centralized testing [28, 29]. Our findings reflect, in part, the smaller capital outlay required for smaller GeneXpert Omni and Edge devices: by enabling flexibility in procuring the number of modules based on testing demands at peripheral centers at approximately one fourth the price of a four-module device, these devices enable smaller peripheral centers to perform point-ofcare testing without a substantive increase in unit costs. However, in settings where the volume of decentralized testing is very low (high costs associated with peripheral testing infrastructure) and an established low-cost sample referral network exists, optimized hub-and-spoke centralized testing is more likely to be cost-effective. 


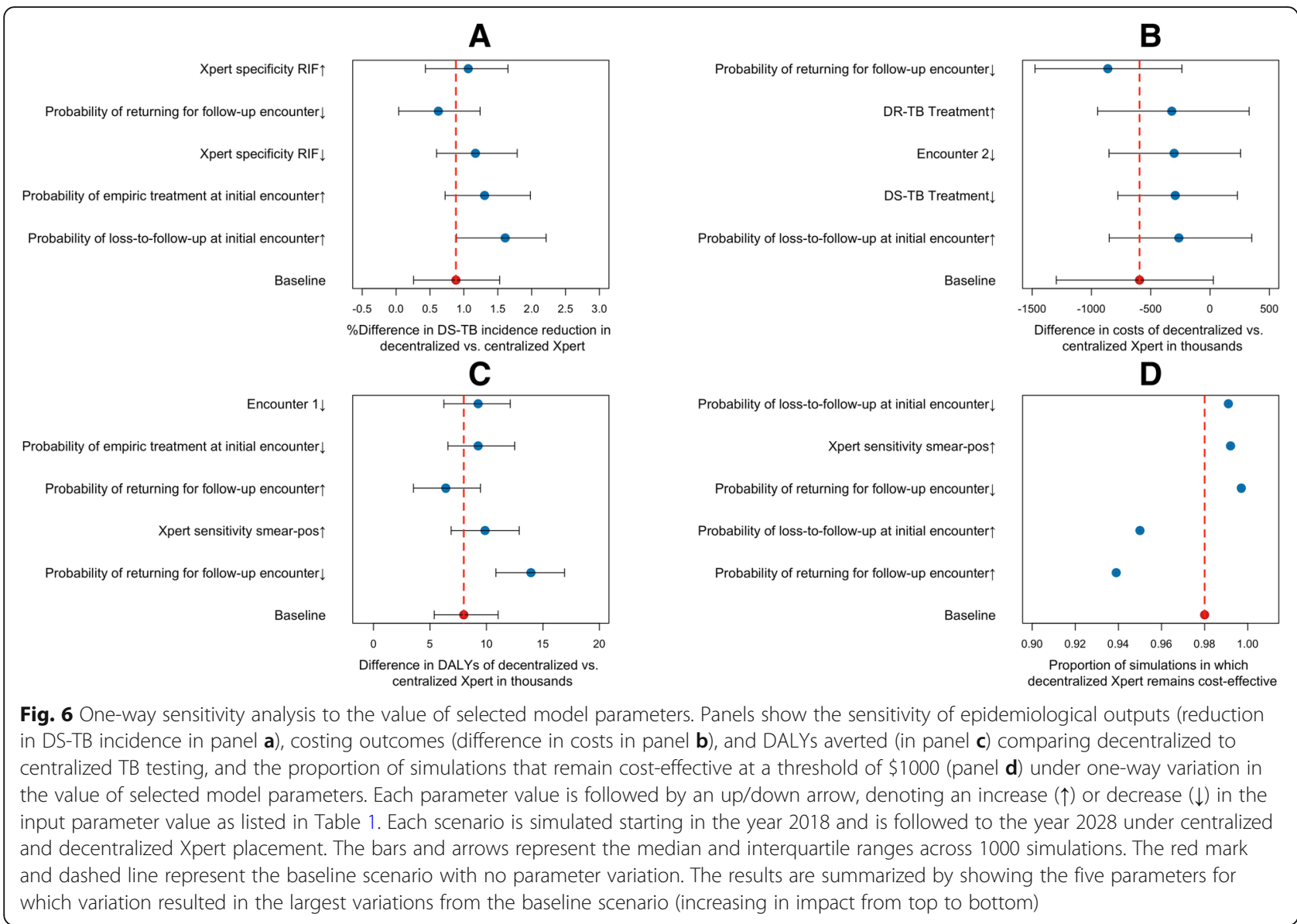

Likewise, it may be financially infeasible to provide peripheral Xpert in all settings (for example, India's 2025 National Strategic Plan [14] aims to provide Xpert in $8335 \mathrm{MCs})$. Thus, our results advocate for prioritizing decentralization of rapid TB CBNAAT in regions with higher-than-average $\mathrm{MC}$ testing volumes and where sample transport systems do not exist for $\mathrm{TB}$ or other diseases while strengthening hub-and-spoke systems (and continuation of smear microscopy) in regions with lower testing volumes in the periphery.

In considering the implementation of molecular diagnostic testing for infectious diseases, it is important to weigh cost-effectiveness alongside considerations of epidemiological impact and feasibility. Our results indicate that the epidemiological impact of decentralized Xpert, while modest, is sufficient to justify its incremental cost in settings characterized by high pre-treatment LTFU, provided that decentralized testing can be feasibly implemented without a loss in quality. However, decentralized testing alone is unlikely to transform TB epidemics in high-burden and heterogeneous settings like India. Therefore, in places where patients are likely to return for follow-up evaluation, parallel investments in strengthening the centralized Xpert system is of equal importance.

Furthermore, with a wide range of novel TB diagnostics (designed for both more peripheral or more centralized placement) [30] approaching market entry, placement strategies for these new technologies will become an important policy debate as countries push to increase the coverage of quality TB diagnosis and care. Our results illustrate that, for technologies optimized for higher level laboratories (e.g., whole-genome sequencing), ability to ensure high testing volumes at the central level and to share health system infrastructure (e.g., specimen transport network) will be important determinants of cost utility. For diagnostics designed for more peripheral placement (e.g., Xpert Edge and Omni, Molbio Truenat MTB), important cost-effectiveness considerations will also include the ability to achieve similar diagnostic performance with simplified logistical infrastructure. As countries plan investments to strengthen the TB care cascade, cost and impact of technologies optimized for different placement strategies must be carefully evaluated against TB control priorities, budget, and current health systems constraints. 
As with any modeling exercise, our study has certain limitations. We adopted a number of simplifying assumptions, including homogeneous mixing, representation of India's TB diagnostic landscape as having only three tiers (informal, formal private, and public), and representation of the diagnostic process as occurring within a discrete number of specific encounters. To the extent that these simplifications depart from the complex reality of TB transmission and diagnosis in the Indian healthcare system, we may over- or underestimate the epidemiological impact and cost-effectiveness of decentralized testing. The most important assumptions in this model are the degree to which decentralized Xpert testing will reduce those who will be permanently lost in the TB care cascade (pre-treatment LTFU, relative to centralized testing) and the degree to which patients will return for follow-up evaluation. Given the complexity of the TB care cascade and patient care-seeking behaviors [31], implementation of decentralized testing alone may not achieve substantive reductions in LTFU [32]. We also did not consider costs of implementing programmatic interventions that may be necessary to translate peripheral testing into reductions in LTFU; where such interventions are required (or where LTFU is already low, even with centralized testing), the cost-effectiveness of decentralized Xpert may be much less favorable than projected here. Nevertheless, we estimated that decentralized testing could be implemented at a lower unit cost than centralized testing in many situations-and to the extent that this can be accomplished (without a loss in quality), decentralized testing is likely to be preferred. Finally, we assumed, for the purpose of comparison, a setting in which Xpert was scaled up as a first-line test for TB among all adults with symptoms; cost-effectiveness of Xpert overall (and of decentralized Xpert relative to centralized Xpert) may be improved by adoption of other algorithms, such as focusing on high-priority populations [33].

\section{Conclusions}

We have used a combined epidemic-economic model to illustrate that decentralized Xpert testing for TB is likely to be a cost-effective strategy in settings characterized by high LTFU, moderate-to-high peripheral testing volumes, and inability to share costs of specimen transport with other disease entities. Our findings reflect the fact that single-module devices enable testing to be performed at lower volumes without considerable increases in per-test costs. Decisions to implement decentralized testing should not be based solely on cost-effectiveness but must also consider budget constraints, feasibility of implementation without a loss in quality, and the degree to which such testing is likely to reduce LTFU in actual practice. Assuming these factors can be addressed, this modeling analysis supports the scale-up of decentralized Xpert testing for $\mathrm{TB}$ as a mechanism to achieve universal access to high-quality $\mathrm{TB}$ diagnosis in India and other similar settings.

\section{Additional file}

Additional file 1: Detailed methodology for cost analyses, simulation model development, and sensitivity analyses. (DOCX 2.10 mb)

\section{Abbreviations}

CBNAAT: Cartridge-based nucleic acid amplification test; CET: Costeffectiveness threshold; DALY: Disability-adjusted life year; DR: Drug resistant; DS: Drug susceptible; DTC: District TB Center; IQR: Interquartile range; LTFU: Loss to follow-up; MC: Microscopy Center; RNTCP: Revised National Tuberculosis Control Program; SSM: Sputum smear microscopy;

TB: Tuberculosis; Xpert: Xpert MTB/RIF

\section{Acknowledgements}

The authors would like to thank Mr. Dean Sher of the SmartSpotQ for sharing cost estimates for external quality control program for Xpert.

\section{Authors' contributions \\ $H S, P K, E K$, and DD were responsible for the conception and design. HS, PK, EK, and DD were responsible for the model development. HS and PK were responsible for the analysis. All authors were responsible for the interpretation and important intellectual input. HS, PK, and DD were responsible for the first draft of the manuscript. HS and PK contributed equally to this work. All authors read and approved the final manuscript.}

\section{Funding}

The study was supported by the TB Modelling and Analysis Consortium (Bill and Melinda Gates Foundation, OPP1083276) and additional support for HS from Fonds de Recherche Santé Post-Doctoral Fellowship training award (36095). The funders of this study had no role or influence in the study design, analysis, or interpretation of the data and in the writing of the manuscript.

\section{Availability of data and materials}

All data generated or analyzed during this study are included in this published article [and its Additional file].

Ethics approval and consent to participate Not applicable

\section{Consent for publication}

Not applicable

\section{Competing interests}

The authors declare that they have no competing interests.

\section{Author details}

${ }^{1}$ Department of Epidemiology, Johns Hopkins Bloomberg School of Public Health, 615 N. Wolfe St., E6529, Baltimore, MD 21205, USA. Division of Infectious Disease, Johns Hopkins University School of Medicine, Baltimore, MD 21205, USA. ${ }^{3}$ Department of Global Health and Development, London School of Hygiene and Tropical Medicine, London WC1E 7HT, UK.

${ }^{4}$ Department of Epidemiology \& Biostatistics \& McGill International TB Centre, McGill University, Montreal, QC H3A 1A2, Canada. ${ }^{5}$ Manipal McGill Centre for Infectious Diseases, Manipal Academy of Higher Education, Manipal, India. 
Received: 22 March 2019 Accepted: 5 July 2019

Published online: 06 August 2019

\section{References}

1. Houben RMGJ, Dodd PJ. The global burden of latent tuberculosis infection: a re-estimation using mathematical modelling. PLoS Med. 2016;13:e1002152.

2. World Health Organization. Global tuberculosis report 2018. 2018.

3. Steingart KR, Schiller I, Horne DJ, Pai M, Boehme CC, Dendukuri N. Xpert $\bullet$ MTB/RIF assay for pulmonary tuberculosis and rifampicin resistance in adults ( review ). Cochrane Libr. 2014. https://doi.org/10.1002/14651858.CD009593. pub3.Copyright.

4. World Health Organization. Xpert meeting report. 2016

5. Vassall A, van Kampen $\mathrm{S}$, Sohn $\mathrm{H}$, et al. Rapid diagnosis of tuberculosis with the Xpert MTB/RIF assay in high burden countries: a cost-effectiveness analysis. PLoS Med. 2011;8:e1001120.

6. Abimbola TO, Marston BJ, Date AA, Blandford JM, Sangrujee N, Wiktor SZ. Cost-effectiveness of tuberculosis diagnostic strategies to reduce early mortality among persons with advanced HIV infection initiating antiretroviral therapy (provisional abstract). J Acquir Immune Defic Syndr. 2012;60:e1-7.

7. Lawn SD, Brooks SV, Kranzer K, Nicol MP, Whitelaw A, Vogt M, Bekker LG, Wood R. Screening for HIV-associated tuberculosis and rifampicin resistance before antiretroviral therapy using the Xpert MTB/RIF assay: a prospective study. PLoS Med. 2011. https://doi.org/10.1371/journal.pmed.1001067.

8. Huddart S, MacLean E, Pai M. Location, location, location: tuberculosis services in highest burden countries. Lancet Glob Heal. 2016:4:e907-8.

9. Raizada N, Sachdeva KS, Sreenivas A, et al. Feasibility of decentralised deployment of Xpert MTB/RIF test at lower level of health system in India. PLoS One. 2014;9:e89301.

10. Macpherson P, Houben MGJ, Glynn JR, Corbett L, Kranzer K. Systematic reviews pre-treatment loss to follow-up in tuberculosis patients in low- and lower-middle-income countries and high-burden countries : a systematic review and meta-analysis. Bull World Health Organ. 2014:126-38.

11. Joloba M, Mwangi $\mathrm{C}$, Alexander $\mathrm{H}$, et al. Strengthening the tuberculosis specimen referral network in Uganda: the role of public-private partnerships. J Infect Dis. 2016;213:S41-6.

12. Vassall A, Siapka M, Foster N, Cunnama L, Ramma L, Fielding K, McCarthy K, Churchyard G, Grant A, Sinanovic E. Cost-effectiveness of Xpert MTB/RIF for tuberculosis diagnosis in South Africa: a real-world cost analysis and economic evaluation. Lancet Glob Heal. 2017;5:e710-9.

13. Dowdy DW. Minding the gap: specimen referral systems for diagnosis of infectious diseases. Clin Infect Dis. 2016;64:ciw820.

14. RNTCP. National strategic plan for tuberculosis elimination. 2017.

15. Kasaie P, Sohn H, Kendall E, Gomez GB, Vassall A, Pai M, Dowdy DW. Exploring the epidemiological impact of universal access to rapid tuberculosis diagnosis using agent-based simulation. In: 2017 Winter Simulation Conference; 2017. p. 1097-108.

16. Sarin S, Huddart S, Raizada N, et al. Cost and operational impact of promoting upfront GeneXpert MTB/RIF test referrals for presumptive pediatric tuberculosis patients in India. PLoS One. 2019:14:e0214675.

17. Xpert Omni fact sheet: MSF concerns on suitability for context. https:// www.ghdonline.org/uploads/OMNI_FACTSHEET_26012018_FINAL_qyHzL8O. pdf. Accessed 06 July 2019

18. Cunnama L, Sinanovic E, Ramma L, et al. Using top-down and bottom-up costing approaches in LMICs: the case for using both to assess the incremental costs of new technologies at scale. Health Econ. 2016;19:1300-17.

19. Bertram MY, Stenberg K, Brindley C, Li J, Serje J, Watts R, Edejer TT-T. Disease control programme support costs: an update of WHO-CHOICE methodology, price databases and quantity assumptions. Cost Eff Resour Alloc. 2017;15:21.

20. Mistry N, Rangan S, Dholakia Y, Lobo E, Shah S. Durations and delays in care seeking, diagnosis and treatment initiation in uncomplicated pulmonary tuberculosis patients in Mumbai, India; 2016. p. 1-17.

21. Salje H, Andrews JR, Deo S, Satyanarayana S, Sun AY, Pai M, Dowdy DW. The importance of implementation strategy in scaling up Xpert MTB/RIF for diagnosis of tuberculosis in the Indian health-care system: a transmission model. PLoS Med. 2014;11:e1001674.

22. Laurence YV, Griffiths UK, Vassall A. Costs to health services and the patient of treating tuberculosis: a systematic literature review. Pharmacoeconomics. 2015;33:939-55.
23. Chatterjee $S$, Levin C, Laxminarayan R. Unit cost of medical services at different hospitals in India. PLoS One. 2013. https://doi.org/10.1371/journal. pone.0069728.

24. Kapoor SK, Raman AV, Sachdeva KS, Satyanarayana S. How did the TB patients reach DOTS services in Delhi? A study of patient treatment seeking behavior. PLoS One. 2012;7:1-6.

25. List of states with population, sex ratio and literacy census 2011. http:// www.census2011.co.in/states.php. Accessed 7 Nov 2017.

26. Bertram MY, Lauer JA, De Joncheere K, Edejer T, Hutubessy R, Kieny P, Hill SR, Bertram MY. Cost - effectiveness thresholds : pros and cons use and misuse of thresholds. Bull World Health Organ. 2016;94:925-30.

27. Woods B, Revill P, Sculpher M, Claxton K. Country-level cost-effectiveness thresholds: initial estimates and the need for further research. Value Health. 2016;19:929-35.

28. Hsiang E, Little KM, Haguma P, Hanrahan CF, Katamba A, Cattamanchi A, Davis JL, Vassall A, Dowdy D. Higher cost of implementing Xpert ${ }^{\oplus}$ MTB/RIF in Ugandan peripheral settings: implications for cost-effectiveness. Int J Tuberc Lung Dis. 2016;20:1212-8.

29. Meyer-Rath G, Schnippel K, Long L, MacLeod W, Sanne I, Stevens W, Pillay S, Pillay $Y$, Rosen $S$. The impact and cost of scaling up genexpert MTB/RIF in South Africa. PLoS One. 2012. https://doi.org/10.1371/journal.pone.0036966.

30. Pai M, Schito M. Tuberculosis diagnostics in 2015: landscape, priorities, needs, and prospects. J Infect Dis. 2015;211:S21-8.

31. Subbaraman R, Nathavitharana RR, Satyanarayana S, Pai M, Thomas BE, Chadha VK, Rade K, Swaminathan S, Mayer KH. The tuberculosis cascade of care in India's public sector: a systematic review and meta-analysis. PLoS Med. 2016;13:1-38.

32. Pai M, Schumacher SG, Abimbola S. Surrogate endpoints in global health research: still searching for killer apps and silver bullets? BMJ Glob Health. 2018;3:e000755.

33. Dowdy DW, Andrews JR, Dodd PJ, Gilman RH. A user-friendly, open-source tool to project impact and cost of diagnostic tests for tuberculosis. Elife. 2014:2014:1-24.

\section{Publisher's Note}

Springer Nature remains neutral with regard to jurisdictional claims in published maps and institutional affiliations.

Ready to submit your research? Choose BMC and benefit from:

- fast, convenient online submission

- thorough peer review by experienced researchers in your field

- rapid publication on acceptance

- support for research data, including large and complex data types

- gold Open Access which fosters wider collaboration and increased citations

- maximum visibility for your research: over $100 \mathrm{M}$ website views per year

At BMC, research is always in progress.

Learn more biomedcentral.com/submissions 